\title{
Influence of Dependence of Directional Extreme Wind Speeds on Wind Load Effects with Various Mean Recurrence Intervals
}

\author{
Xinxin Zhang ${ }^{1}$, Xinzhong Chen $^{2}$ \\ ${ }^{1} \mathrm{PhD},{ }^{2}$ Professor, National Wind Institute, Department of Civil, Environmental, and Construction \\ Engineering, Texas Tech University, Lubbock, TX 79409, USA.
}

\begin{abstract}
This study offers an improved understanding of the influence of statistical dependence between directional extreme wind speeds when estimating the wind load effects with various mean recurrence intervals (MRIs). Existing multivariate approaches that concern the wind directionality effect are first reviewed. Several factors that influence the prediction with and without consideration of the statistical dependence between directional extreme wind speeds are discussed by using Gaussian copula model. The influence of wind speed masking on the wind effect estimation is discussed. The influence of use of different joint probability distribution models for directional extreme wind speeds is illustrated through a comparison between multivariate Gaussian and Gumbel copula models. The necessity of using multivariate approach is discussed and a simplified method is proposed to account for directional dependence, which not only provides accurate prediction but also reduces calculation effort. Examples with real wind climate model and generic wind tunnel test results are shown to illustrate the influences brought by directional dependence, model difference, and wind speed masking. Also discussion is made on the partition of directional sectors which concerns the balance of number of sectors and modeling uncertainty.
\end{abstract}

Key words: Wind effect, Directionality, Gaussian copula, Multivariate Gumbel copula, Directional wind speed dependence, Wind speed masking. 


\section{Introduction}

Estimation of wind load effects with various mean recurrence intervals (MRIs) requires

27 consideration of uncertainty and directionality of wind climate, aerodynamics and structural dynamics.

28 Without consideration of directionality, probabilistic methods have been developed to integrate the

29 probability distributions of yearly maximum wind speed and wind load effect (response) conditional on

30 wind speed (Cook and Mayne 1979, 1980; Harris 1982 and 2005; Chen and Huang 2010). Other

31 probabilistic models have also been addressed in literature to account for various uncertainties (e.g.,

32 Kareem 1987, 1988 and 1990; Diniz et al. 2004; Diniz and Simiu 2005; Hanzlik et al. 2005). On the other

33 hand, to address the directionality effects of wind climate, aerodynamic and structural dynamics, several

34 approaches have been developed. Some of these approaches are based on parent distribution of wind

35 speed (e.g., Davenport 1977; Lepage and Irwin 1985; Irwin et al. 2005), and the others on extreme wind speed data or wind storm passage data (e.g., Simiu and Filliben 1981; Simiu and Heckert 1998, Isyumov

37 et al. 2003 and 2014). All these approaches treat the wind load effect conditional on wind speed and direction as a deterministic quantity. Furthermore, there is a lack of consensus in the estimated design wind loads and responses for specified MRIs using different methods given that the dynamic wind loads derived by different experienced laboratories are in general very consistent. For instance, the wind-

41 induced responses of the World Trade Center (WTC) towers estimated by two recent independent studies

42 from two world leading wind engineering laboratories in North America differ from each other by about $4340 \%$ - difficult for many structural engineers to accept as normal variability (Sadek 2005). reduction from the worst case estimation (e.g., ASCE 2010). However, the directionality factor is generally associated with many other factors such as characteristics of directional wind speed and 47 aerodynamics, and the MRI of target response. The adequacy of the directionality factor specified in 48 design standards has been reexamined in literature (Simiu and Heckert 1998; Rigato et al. 2001; Laboy49 Rodriguez et al. 2014; Isyumov et al. 2014; Zhang and Chen 2015). 
Recently, Zhang and Chen (2015) introduced a unified approach for estimating probabilistic wind

51 effect with consideration of both directionality and uncertainty, where the joint probability distribution of

52 directional extreme wind speeds was modeled by using multivariate extreme value theory with Gaussian

53 copula. The Gaussian copula is the same as the multivariate Gaussian translation model (Grigoriu 2007

54 and 2009), which was used as a basis for generating directional extreme wind speeds (Yeo 2014).

55 Multivariate extreme wind speed models have also been addressed in literature in terms of bivariate

56 Gumbel distribution model (Simiu et al. 1985), and multivariate Gumbel distribution model (Itoi and

57 Kanda 2002). It is reported that during a synoptic storm passage, the wind direction may vary about 200

58 degrees on average (e.g., Cook 1982). Therefore, the extreme wind speeds in neighboring sectors often

59 have certain level of correlation. The conservatism of using the independent assumption was proved and

60 the underestimate from fully correlated assumption was also recognized (Simiu et al. 1985; Irwin et al.

$612005)$.

62 This study addresses a number of issues related to the modeling of multivariate distribution of 63 directional extreme wind speeds and its influence on the predicted wind effects with various MRIs. The

64 influence of correlation coefficient between directional extreme wind speeds on the estimated wind

65 effects is investigated. The results from the two variants of sector-by-sector method that assume full-

66 correlation and independence of directional wind speeds are compared. Due to current data recording and

67 analysis mechanism, some directional wind speed data are often masked (Coles and Walshaw 1994, Vega

68 2008). The masking issue affects not only the statistics of directional wind speeds but also their

69 dependence. This study further discusses the influence of masking issue on the predicted wind load

70 effects. A simplified procedure with consideration of the dependence of only a small number of important

71 directional wind speeds is proposed for computational efficiency. This study also compares the

72 multivariate Gumbel and Gaussian copula models which sheds insight on the model selection. Finally,this

73 study examines the influence of the partition number of directional wind speeds on the prediction, which

74 provides information on the modeling uncertainty. 


\section{$77 \quad 2.1 \quad$ Approaches based on multivariate directional extreme wind speed model}

The probabilistic extreme response for the $i$-th wind direction $(i=1,2, \ldots, n)$ with a given time duration, say, one hour, is often expressed as a function of mean wind speed:

$$
X_{i}=\frac{1}{2} \rho V_{i}^{2} C_{i}\left(V_{i}\right)
$$

$$
C_{i}\left(v_{i}\right)=v_{i}^{b-2} C_{0 i} c_{\alpha i}
$$

82 where $C_{0 i}$ is generally a random quantity with a unit mean, which reflects the uncertainty of extreme

83 response coefficient in the $i$-th direction; $c_{\alpha i}$ is a deterministic value for the $i$-th direction, which reflects

84 the directionality characteristics of extreme response coefficient, and $\max \left\{c_{\alpha i}, i=1,2, \ldots, n\right\}=1 ; b$ is a

85 power law exponent indicating the growth rate of the extreme response with wind speed. In the case of rigid structures, the wind load coefficient $C_{i}\left(v_{i}\right)$ can be independent of mean wind speed, i.e., $b=2$. On

87 the other hand, it is a function of mean wind speed for flexible structures due to dynamic amplification effect, i.e., $b>2$.

When extreme response conditional on given wind speed and direction is considered as a deterministic quantity, i.e., $C_{0 i}$ is deterministic, the cumulative distribution function (CDF) of extreme response considering directionality can be determined as:

$$
\begin{aligned}
\Psi_{X}(x)=P\left(X_{1}\right. & \left.\leq x, X_{2} \leq x, \ldots, X_{n} \leq x\right)=P\left(V_{1} \leq v_{x, 1}, V_{2} \leq v_{x, 2}, \ldots, V_{n} \leq v_{x, n}\right) \\
& =H\left(v_{x, 1}, v_{x, 2}, \ldots, v_{x, n}\right)
\end{aligned}
$$

92 where $x$ is a given wind effect level; $v_{x, i}=\sqrt{2 x / \rho C_{i}\left(v_{x, i}\right)}$ is the corresponding wind speed in the $i$-th 93 direction causing response level $x ; H\left(v_{1}, v_{2}, \ldots, v_{n}\right)$ is the joint CDF (JCDF) of directional extreme wind 94 speeds. 
When $C_{0 i}$ thus $C_{i}$ is a random variable, under the assumption that largest extreme response in a year for a given wind direction is always the result of the strongest wind speed (Cook and Mayne 1979, 1980),

97 Eq. (3) becomes (Zhang and Chen 2015):

$$
\Psi_{X}(x)=\int \ldots \int \mathrm{H}\left(v_{x 1}\left|c_{1}, \ldots, v_{x n}\right| c_{n}\right) f\left(c_{1}, \ldots, c_{n}\right) d c_{1} \ldots d c_{n}
$$

where $f\left(c_{1}, \ldots, c_{n}\right)$ is the joint probability density function (JPDF) of $C_{i}\left(V_{i}\right)(i=1,2, \ldots, n)$. In general, $C_{i}\left(V_{i}\right)(i=1,2, \ldots, n)$ are mutually independent, thus $f\left(c_{1}, \ldots, c_{n}\right)=f_{1}\left(c_{1}\right) f_{2}\left(c_{2}\right) \ldots f_{n}\left(c_{n}\right)$, where $f_{i}\left(c_{i}\right)$ is the PDF of $C_{i}\left(V_{i}\right)$. Technically, Eq.(4) can be evaluated through Monte Carlo simulation, i.e., by first generating samples of $c_{i}\left(v_{i}\right)=c_{i}(i=1,2, \ldots, n)$, and then calculating $\mathrm{H}\left(v_{x_{1}}\left|c_{1}, \ldots, v_{x n}\right| c_{n}\right)$, followed by ensemble average of these estimations. From $\Psi_{X}(x)$, the wind load effect for MRI $=\mathrm{R}$ year, $x_{\mathrm{R}}$, can be determined as $\Psi_{X}\left(x_{\mathrm{R}}\right)=1-1 / R$. It is noted that Eq.(4) can also be given in terms of JCDF of $C_{i}\left(V_{i}\right)(i=1,2, \ldots, n)$ and JPDF of directional extreme wind speeds, which can be computationally more efficient through Monte Carlo simulation when $C_{i}\left(V_{i}\right)(i=1,2, \ldots, n)$ are mutually independent (Zhang and Chen 2015).

It should be noted that the full-order method (Harris 1982 and 2005; Chen and Huang 2010), which accounts for the probability of the largest wind effect in a year for a given wind direction caused by second or high-order strongest wind speeds, results in improved estimations of wind effects with relatively lower MRIs as compared to the first-order method introduced by Cook and Mayne $(1979,1980)$. As pointed in Chen and Huang (2010), such an improvement is negligibly small for wind effects with large MRIs. Therefore, Eq.(4) is considered as adequate to address both directionality and uncertainty when the focus is placed on the wind effects with large MRIs.

The above approach combines the consideration of directionality and uncertainty in a unified framework with an analytical formulation which facilitates a systematic parametric study. Moreover, the work of statistical modeling of wind climate information, i.e., determination of $H\left(v_{1}, v_{2}, \ldots, v_{n}\right)$ and the work of wind tunnel testing, i.e., the determination of $C_{i}\left(v_{i}\right)$, can be separately conducted yet integrated through this framework. Also, the use of statistical model of directional extreme wind speeds makes the 
estimation for multiple responses under the same wind climate condition more efficient by avoiding the interpretation of extreme wind effect samples from the original wind speed data.

121

122

123

124

125

126

127

128

129

130

131

132

133

134

135

136

137

138

139

\subsection{Modeling of multivariate directional extreme wind speeds}

As introduced previously, now the problem of estimating the probabilistic wind load effect with consideration of directionality is converted to the modeling of the JCDF of directional extreme wind speeds which falls into the concerns of multivariate extreme value theory. For any multivariate extreme problem, the first step is to determine the extreme value distributions of univariate variables, i.e., marginal distributions. The extreme wind speed in the $i$-th sector is assumed to follow Gumbel (Type I) distribution and its CDF is expressed as:

$$
F_{i}\left(v_{i}\right)=\exp \left[-\exp \left(-\frac{v_{i}-m_{i}}{\delta_{i}}\right)\right]
$$

where $m_{i}$ and $\delta_{i}$ are location and scale parameters. The mean and standard deviation (STD) of the Type I distribution are $m_{i}+\gamma \delta_{i}$ and $\delta_{i} \pi / \sqrt{6}$, respectively, where $\gamma=0.5772$ is Euler constant.

The multivariate random variables $V_{1}, V_{2}, \ldots, V_{n}$ can be then transformed into random variables $U_{1}, U_{2}, \ldots, U_{n}$ which follow uniform distributions on domain $[0,1]$ through the mapping of CDF functions, i.e., $u_{i}=F_{i}\left(v_{i}\right),(i=1,2, \ldots n)$. It can be proved that there is always a unique function, $C_{\text {copula }}($.$) ,$ called copula, that relates the standard margins, if they are continuous, to the JCDF as (Nelsen, 2006):

$$
H\left(v_{1}, v_{2}, \ldots, v_{n}\right)=C_{\text {copula }}\left(F_{1}\left(v_{1}\right), F_{2}\left(v_{2}\right), \ldots, F_{n}\left(v_{n}\right)\right)=C_{\text {copula }}\left(u_{1}, u_{2}, \ldots, u_{n}\right)
$$

A copula represents a multivariate probability distribution for which the marginal probability distribution of each variable is uniform. Copulas are used to describe the statistical dependence between random variables. Their name comes from the Latin for "link" or "tie".

\subsubsection{Gaussian copula model}

The Gaussian copula is one of the many copulas and is defined using the multivariate Gaussian distribution as follows: 


$$
C_{\text {copula }}\left(u_{1}, u_{2}, \ldots, u_{n}\right)=G_{n}\left(\Phi^{-1}\left(u_{i}\right), \Phi^{-1}\left(u_{2}\right), \ldots, \Phi^{-1}\left(u_{n}\right)\right)
$$

where $\Phi$ is the $\mathrm{CDF}$ of standard Gaussain distribution; and $G_{n}$ is the $\mathrm{CDF}$ of $n$-dimensional Gaussian distribution with mean 0 and covariance matrix $\Sigma$ where $\Sigma_{i j}=\Sigma_{j i}=\rho_{i j}=E\left[\Phi^{-1}\left(U_{i}\right), \Phi^{-1}\left(U_{j}\right)\right]$. Therefore, the JCDF of directional extreme wind speeds can be derived by combining Eq. (6) and (7) as:

$$
H\left(v_{1}, v_{2}, \ldots, v_{n}\right)=G_{n}\left(y_{1}, y_{2}, \ldots y_{n}\right)
$$

where $y_{i}=\Phi^{-1}\left(u_{i}\right)=\Phi^{-1}\left(F_{i}\left(v_{i}\right)\right)=g_{i}\left(v_{i}\right)$ is the value of underlying Gaussian variable $Y_{i}$; and $g_{i}$ is the translation function for $V_{i}$. The CFD of extreme wind speed regardless of wind direction can be then determined as $\Psi_{V}(v)=H(v, v, \ldots, v)$, and the probabilistic wind load effect can be determined from Eq. (3) or (4).

The Gaussian copula model captures the dependence structure in a straightforward manner through the multivariate Gaussian distribution in conjunction with its covariance matrix, which is naturally applicable to more than two variables. However, the computational effort may be considerable especially when more than four variables are included for an accurate estimation of their JCDF (Genz and Bretz 2002).

\subsubsection{Multivariate Gumbel copula and HK model}

One of the widely used copula families is Archimedean copula family which includes various members but can all be expressed in a general format below (Nelson 2006):

$$
C_{\text {copula }}\left(u_{1}, u_{2}\right)=\varphi^{-1}\left(\varphi\left(u_{1}\right)+\varphi\left(u_{2}\right)\right)
$$

where $\varphi(t), t \in[0,1]$, is called generator of the copula which is a continuous, strictly decreasing function and must satisfy $\varphi(1)=0$. When $\varphi(t)=(-\ln t)^{\theta}$, the copula returns Gumbel copula which is also known as Gumbel's bivariate distribution. If the directional extreme wind speed follows Gumbel distribution, the JCDF can be expressed as:

$$
H\left(v_{1}, v_{2}\right)=\exp \left\{-\left[\mathrm{e}^{\frac{-\theta\left(v_{1}-m_{1}\right)}{\delta_{1}}}+\mathrm{e}^{\frac{-\theta\left(v_{2}-m_{2}\right)}{\delta_{2}}}\right]^{\frac{1}{\theta}}\right\}
$$


where $\theta=\left(1-\rho_{12}\right)^{-1 / 2}$ represent the dependence; and $\rho_{12}$ is the correlation coefficient of $V_{1}$ and $V_{2}$.

One of the extensions to multivariate Archimedean copula has the following form:

$$
C_{\text {copula }}^{n}\left(u_{1}, u_{2}, \ldots, u_{n}\right)=\varphi^{-1}\left(\varphi\left(u_{1}\right)+\varphi\left(u_{2}\right)+\cdots+\varphi\left(u_{\mathrm{n}}\right)\right)
$$

Although such an extension may not be a true copula for many Archimedean copulas, it is a true copula for Gumbel copula (Nelsen 2006). The drawback of this extension is that it offers only one parameter $\theta$ for a multi-dimensional space, which, however, is most likely not sufficient to represent the complication of the full dependence of the multivariate extremes such as the directional extreme wind speeds (Haraguchi and Kanda 1999).

Another way to extend a bivariate copula to a multivariate copula is based on the claim that for an $n$ dimensional Archimedean copula, it is the serial iterates of the bivariate Archimedean copula (Nelsen 2006, Schweizer and Sklar 1983) and has a relationship with its $n-1$ dimensional Archimedean copula:

$$
C_{\text {copula }}^{n}\left(u_{1}, u_{2}, \ldots, u_{n}\right)=C_{\text {copula }}\left(C_{\text {copula }}^{n-1}\left(u_{1}, u_{2}, \ldots, u_{n-1}\right), u_{n}\right)
$$

Unfortunately, the construction of such a multivariate copula generally fails except for some particular cases. Nevertheless, this technique of constructing a multivariate copula was used for directional wind speed model by Haraguchi and Kanda (1999), referred to as HK model, where the scale parameters of Gumbel distribution for wind speed of each direction is assumed to identical, i.e., $\delta_{i}=\delta_{j}$ for all $i, j=1,2, \ldots, n$ and $i \neq j$ (Itoi and Kanda 2002). In the HK model, the joint CDF of directional wind speeds for four directions was proposed based on Eq. (12) and extended from Eq. (10) as:

$$
\begin{aligned}
& H\left(v_{1}, v_{2}, v_{3}, v_{4}\right) \\
& =\exp \left[-\left[\left\{\left[\mathrm{e}^{\frac{-\theta_{1}\left(v_{1}-m_{1}\right)}{\delta_{1}}}+\mathrm{e}^{\frac{-\theta_{1}\left(v_{2}-m_{2}\right)}{\delta_{2}}}\right]^{\frac{\theta_{2}}{\theta_{1}}}+\mathrm{e}^{\frac{-\theta_{2}\left(v_{3}-m_{3}\right)}{\delta_{3}}}\right\}^{\frac{\theta_{3}}{\theta_{2}}}+\mathrm{e}^{\frac{-\theta_{3}\left(v_{4}-m_{4}\right)}{\delta_{4}}}\right)^{\frac{1}{\theta_{3}}}\right]
\end{aligned}
$$

where $V_{1}, V_{2}, V_{3}, V_{4}$ are sorted in the order of dominance, i.e., $V_{1}$ and $V_{4}$ are wind speeds of the most and the least dominant directions respectively, and $\delta_{1}=\delta_{2}=\delta_{3}=\delta_{4}=\delta ; \theta_{1}, \theta_{2}$ and $\theta_{3}$ represent the dependence of each iterate and are determined as: 


$$
\theta_{1}=\left(1-\rho_{\theta_{1}}\right)^{-1 / 2}, \theta_{2}=\left(1-\rho_{\theta_{2}}\right)^{-1 / 2}, \quad \theta_{3}=\left(1-\rho_{\theta_{3}}\right)^{-1 / 2}
$$

178 where $\rho_{\theta_{1}}, \rho_{\theta_{2}}$ and $\rho_{\theta_{3}}$ are determined empirically as (Itoi and Kanda 2002):

$$
\begin{aligned}
& \rho_{\theta_{1}}=\rho_{12} \\
& \rho_{\theta_{2}}=\frac{\Delta y_{13} \rho_{13}+\Delta y_{23} \rho_{23}}{\Delta y_{13}+\Delta y_{23}} \\
& \rho_{\theta_{3}}=\frac{\Delta y_{14} \rho_{14}+\Delta y_{24} \rho_{24}+\Delta y_{34} \rho_{34}}{\Delta y_{14}+\Delta y_{24}+\Delta y_{34}} \\
& \Delta y_{i j}=\frac{m_{i}-m_{j}}{\delta}
\end{aligned}
$$

When directional wind speeds are assumed to be independent, i.e., $\rho_{i j}$ for all directions are 0, Eq. (13) becomes $H\left(v_{1}, v_{2}, v_{3}, v_{4}\right)=\prod_{i=1}^{4} F_{i}\left(v_{i}\right)$.

This model is proved to be consistent when the assumption of identical scale parameter for each variable is satisfied. Practically, the directional wind speeds generally have different scale parameters so that a change of variable is needed when using the HK model. New variables $V_{i}^{\prime}=V_{i} / \delta_{i}(i=1,2,3,4)$ are introduced, which follow Gumbel distributions as $F_{V_{i}^{\prime}}\left(v_{i}^{\prime}\right)=\exp \left[-\exp \left(-\left(v_{i}^{\prime}-m_{i}^{\prime}\right)\right)\right]$, where $m_{i}^{\prime}=m_{i} / \delta_{i}$. These new variables have the identical scale parameter of one, thus the HK model can be used to describe their JCDF denoted as $H^{\prime}\left(v_{1}^{\prime}, v_{2}^{\prime}, v^{\prime}{ }_{3}, v^{\prime}{ }_{4}\right)$. The CFD of extreme wind speed regardless of wind direction can be then determined as $\Psi_{V}(v)=H^{\prime}\left(v / \delta_{1}, v / \delta_{2}, v / \delta_{3}, v / \delta_{4}\right)$. Further following Eq. (4), estimation of directional wind effect can be made as $\Psi_{X}(x)=H^{\prime}\left(v_{x 1} / \delta_{1}, v_{x 2} / \delta_{2}, v_{x 3} / \delta_{3}, v_{x 4} / \delta_{4}\right)$. While the extension of the HK model to more than four variables is possible, the corresponding equation for replacing Eq.(15) becomes very complex thus is not discussed here.

It is clear that both multivariate Gaussian and Gumbel copulas contain dependence parameters with their respective analytical formulations, i.e., covariance matrix $\Sigma$ in Gaussian copula and $\theta_{1}, \theta_{2}$ and $\theta_{3}$ in Gumbel copula. Taking such an advantage, these two multivariate wind speed models are not only able to account for the influences brought by the statistical dependence of directional wind speeds but also offer analytical tools for parametric studies. 


\subsection{Sector-by-sector method}

The sector-by-sector (SBS) method is also often used due to its simplicity (Simiu and Filliben 2005; Irwin et al. 2005; Holmes 2001). It determines the extreme response for a target MRI directly from the extreme wind speeds at different directions. Two variants of this approach are adopted in practice: 1) The wind speeds at various directions (sectors) with a target MRI of R years are firstly determined. The corresponding extreme responses are quantified and their maximum over all sectors is considered as the response for MRI of $\mathrm{R}$ years; 2) The CDFs of annual maximum responses at different sectors are determined from the yearly extreme value distributions of directional wind speeds. The CDF of annual maximum response over all directions is then estimated as a product of these CDFs of directional extremes under the assumption that directional extreme wind speeds are mutually independent. Both predictions can be less accurate as the statistical dependence of directional extreme wind speeds is not adequately accounted. The performance of first variant of SBS method (SBS1) was addressed in Simiu and Filliben (2005).

Analytically, when all the directional wind speeds are fully correlated, Eq. (4) becomes

$$
\Psi_{X}(x)=P\left(V_{1} \leq v_{x, 1}, V_{2} \leq v_{x, 2}, \ldots, V_{n} \leq v_{x, n}\right)=P\left(V_{k} \leq v_{x, k}\right)=F_{k}\left(v_{x, k}\right)
$$

210 where $k$ denotes the direction with $F_{k}\left(v_{x, k}\right) \leq F_{j}\left(v_{x, j}\right)(j=1,2, \ldots n, j \neq k)$. Clearly, when the $R$-year 211 wind effect, $x_{R}$, is considered, the corresponding wind speed $v_{x, k}$ is the R-year wind speed in the $k$-th 212 direction, i.e., $v_{x, k}=v_{R, k}$, while $v_{x, k}$ is not less than the R-year wind speed in the $j$-th direction, i.e, $213 v_{x, j} \leq v_{R, j}$. In other words, the wind effect under R-year wind speed in the $j$-th direction is not larger 214 than $x_{R}$. In such a fully correlated case, the first variant (SBS1) results in accurate prediction (Simiu and 215 Filliben 2005), and the wind effect of MRI = R year can be expressed as:

$$
x_{\mathrm{R}}=\frac{1}{2} \rho v_{R, k}^{2} C_{k}\left(v_{R, k}\right)
$$

When all the directional wind speeds are assumed to be independent, Eq. (4) becomes 


$$
\Psi_{X}(x)=P\left(V_{1} \leq v_{x, 1}, V_{2} \leq v_{x, 2}, \ldots, V_{n} \leq v_{x, n}\right)=\prod_{i=1}^{\mathrm{n}} P\left(V_{i} \leq v_{x, i}\right)=\prod_{i=1}^{\mathrm{n}} F_{i}\left(v_{x, i}\right)
$$

Eq. (18) corresponds to the second variant of SBS method (SBS2). It is proved that Eq. (16) or (17) usually leads to an underestimate of wind effect unless the worst case scenario occurs, i.e., the directions of strongest wind speed and largest wind load effect coefficient coincide. On the other hand, Eq. (18) is considered to be conservative when correlation coefficients of directional wind speeds are non-negative (Irwin et al. 2005; Simiu and Filliben 2005). It is also proved that Eq. (18) has negligible difference to that estimated under negative correlations (Zhang and Chen 2015).

Although both SBS methods are incapable of accounting for the directional dependence, they do offer a good judgment of the region where the true estimate should fall into. In fact, assuming all the directional extreme wind speeds are non-negatively correlated, the estimates from SBS1 and SBS2 construct the lower and upper bounds respectively (Nelsen 2006; Grigoriu 2007). This is insured by one of the most important properties of a multivariate distribution that, take Gaussian copula for an example, $H\left(v_{1}, v_{2}, \ldots, v_{n} ; \Sigma\right) \geq H^{\prime}\left(v_{1}, v_{2}, \ldots, v_{n} ; \Sigma^{\prime}\right)$ if $\Sigma_{i j} \geq \Sigma^{\prime}{ }_{i j}$ for any $i \neq j$.

\section{Influence of correlation between directional extreme wind speed}

The multivariate approach provides an opportunity to investigate and quantify the influence of correlation of directional extreme wind speeds on the predictions. Previous studies show that when the correlation is not strong, say, the correlation coefficient is less than 0.7 , the consideration of correlation does not make noticeable influence on the predictions compared to independent assumption (Grigoriu 2009). In the following, the influence of correlation of directional extreme wind speeds is further discussed based on Gaussian copula model.

In the case where there is only one dominant direction, the overall wind effect is largely determined by this dominant direction and the other directions have minimum contributions. Supposed that the $k$-th direction is the dominant direction among $n$ directions, for a given wind effect $x$, the probability of not exceeding $x$ in the $k$-th direction is $F_{k}(x)$ and for any $i$-th direction, $F_{i}(x) \approx 1$ and $F_{k}(x)<F_{i}(x)$ 
$240(i \leq n, i \neq k)$. The lower bound CDF calculated from $\operatorname{SBS} 1$ is $\Psi_{X, S B S 1}(x)=F_{k}(x)$. The upper bound

$241 \mathrm{CDF}$ calculated from SBS2 is $\Psi_{X, S B S 2}(x)=F_{k}(x) \prod_{i=1, i \neq k}^{n} F_{i}(x) \approx F_{k}(x)=\Psi_{X, S B S 1}(x)$. Because the 242 calculation that accounts for the directional dependence must lie in between these two bounds, it indicates 243 that the consideration of directional dependence has no significant influence.

247 Gumbel distribution, and the wind load effect coefficients at these two directions are also identical and deterministic. The joint distribution of extreme wind speeds is described by Gaussian copula with a correlation coefficient $\rho_{i j}$. The R-year underlying Gaussian variable $y_{R}$, the corresponding wind speed $v_{R}$ 250 and wind effect $x_{R}$ are determined as

$$
\begin{gathered}
G_{2}\left(y_{R}, y_{R} ; \rho_{i j}\right)=1-1 / R \\
x_{R}=\frac{1}{2} \rho v_{R}^{b} C\left(v_{R}\right) \\
\left.v_{R}=\mu_{v}\left(1+V_{v} \frac{\sqrt{6}}{\pi}-\ln \left\{-\ln \left[\Phi\left(y_{R}\right)\right]\right\}\right)+\gamma\right)
\end{gathered}
$$

where $G_{2}\left(y_{R}, y_{R}\right)$ is bivariate Gaussian $\mathrm{CDF}$ with correlation coefficient $\rho_{i j} ; \mu_{v}$ and $V_{v}$ are mean and coefficient of variation for the directional wind speeds.

Denotes $y_{R}^{\prime}$ as the value of $y_{R}$ when $\rho_{i j}=0$. According to the properties of multivariate Gaussian distribution, i.e., when $\rho_{i j} \geq 0, G_{2}\left(y_{R}, y_{R} ; \rho_{i j}\right) \geq G_{2}\left(y_{R}, y_{R} ; 0\right)$, we have $y_{R} \leq y_{R}^{\prime}$ because $G_{n}$ is monotonically increasing in all dimensions (Nelsen 2006). Fig.1 shows the influence of the correlation coefficient on the predictions in terms of $\delta y=y_{R}^{\prime} / y_{R}-1$ for $\mathrm{R}=10,50$ and 500 years, respectively. It is observed that the difference of predictions with and without consideration of correlation grows exponentially when the correlation coefficient increases. On the other hand, the difference reduces for a larger MRI. 
The difference in the predicted wind effects can be subsequently quantified in terms of $\delta x=$ $x_{R}^{\prime} / x_{R}-1$. From Eq. (19), it is noted that the difference is affected only by the coefficient of variation of extreme wind speed $V_{v}$ and the dynamic sensitivity parameter $b$ as shown in Fig.2. The difference $\delta x$ is larger when $V_{v}$, is larger, and is more significant for a flexible structure $(\mathrm{b}>2)$ than for a rigid structure.

264 For $\mathrm{R}=50$ years with $\rho_{i j}=0.9$, when $V_{v}$ increases from 0.2 to $0.3, \delta x$ increases from $6.5 \%$ to $8.3 \%$ for $\mathrm{b}$ $265=2$, and from $10.0 \%$ to $12.7 \%$ for $\mathrm{b}=3$. The difference reduces for wind effect with a larger MRI. For 266 example, for $\mathrm{R}=500$ years with $\rho_{i j}=0.9$, in the case $V_{v}=0.2, \delta x_{R}=4.1 \%$ when $b=2$, and $\delta x_{R}=6.3 \%$ 267 when $b=3$.

The above discussions are all based on bivariate cases. It should be noted that when the number of correlated variables, $n$, increases, the impact from the correlation will accumulate. Intuitively, we calculated the case where $n=3$ and the covariance matrix has a same value for all off diagonal terms, i.e., 271 each two directions has the same correlation coefficient $\rho_{i j}$. Fig. 3 shows the comparison of $n=2$ and $n=$ 2723 for $\mathrm{R}=50$ and 500 years when $V_{v}=0.3$. In all cases, adding more correlated variables will add to the 273 magnitude of $\delta x_{R}$, which means the consideration of directional dependence is more significant when 274 more dominant directions exist. For example, when MRI $=50$ years, $b=3$ and $\rho_{i j}=0.9, \delta x_{R}$ increases 275 from $12.7 \%$ to $20.8 \%$ when the correlated variables changed from $n=2$ to 3 . The discussion using 276 realistic wind speed and wind load effect models will be addressed in the later part of this study

\section{4. Influence of directional wind speed masking}

Due to the data recording mechanism and analysis method, e.g., wind speed data post-processing, the continuous hourly mean wind speed and direction data may not be available at a given site of interest. For 280 instance, only the maximum hourly mean wind speed and direction over a given period of time, say, 3 281 hours or 24 hours (daily), may be reported. When the analysis is based on these discontinuous data, the 282 relative large wind speeds at directions different from the one that is reported are masked. The directional 283 wind speed masking problem has been discussed in literature (e.g., Cook 1982; Moriarty and Templeton 
1983). Although a complete treatment of masking problem requires high resolution data of a particular site of interest, which often leads one to resort to the original wind speed data if available, a simple yet arbitrary procedure was introduced to treat the masking problem by using component wind speeds to represent the wind speed in the masked directions (Coles and Walshaw 1994). Let $v_{\phi}$ denote the magnitude of wind speed in direction $\phi$ which is recorded, the masked wind speed in direction $\alpha$ over the same time duration is estimated as a component of the recorded wind speed: $v_{\alpha}=v_{\phi} \cos (\alpha-\phi)$ if $|\alpha-\phi|<\pi / 2$. Wind speeds in directions outside of this range will be considered as 0 . Applications of this procedure have been seen in literature (e.g., Payer and Küchenhoff 2004; Vega 2008).

This study uses the directional hourly mean wind speed record at Baltimore, MD, USA, dated from January $1^{\text {st }}, 2000$ to August $31^{\text {st }}, 2012$, in which 16 directional sectors were divided evenly and represented by their center directions, i.e., E, ENE, ..., ESE. It should be noted that 2 months of data are within the impact of hurricanes and therefore deliberately dropped. Therefore, there are 150 months of continuous directional hourly wind speeds which are referred to as the "full rank" data in this study. In order to demonstrate the influence of the masking issue, two sets of "masked" data are generated by choosing one maximum hourly mean wind speed for each 3-hour interval and 24-hour interval (daily maxima), respectively. These three sets of data are analyzed using the same framework and the results are compared.

The monthly maximum wind speeds at each direction are fitted by Gumbel distribution and the predicted 50- and 500-year wind speeds are summarized in Fig. 4. It is observed that there is only minor influence from the masking issue since the predictions from full rank and masked data are close in every direction. This means the marginal distributions for each of the data set are consistent. Further, the Gaussian copula model is used to calculate the joint distribution of directional extreme wind speeds, from which the 50- and 500-year non-directional wind speed can be determined. The correlation coefficient matrix for the full rank data can be found in Table 1. There is no obvious differences changes between 

presents consistent results. Also, since all the maxima of the non-directional wind speeds are kept the same for full rank and masked data, the predictions by directly fitting the Gumbel distribution to the non-

312 directional data are identical. It is noted that the estimates from Gaussian copula model are generally 313 consistent with the results from direct fit to the non-directional data but slight difference exists due to the 314 modeling uncertainties (Zhang and Chen 2015).

Since the masking issue does not affect the modeling of directional extreme wind speeds much, it should have minimum influence on the estimation of extreme wind effect. To demonstrate this point, two 317 examples of extreme wind effect coefficient were used, i.e., Examples 1 and 2, as shown in Fig. 5. These two wind load coefficient examples was generated based on the directionality characteristics of some of the generic typical responses of wind-excited tall buildings (Isyumov et al. 2014). The wind effects calculated with both masked and full rank wind speed data are compared. Fig. 6 shows the comparison of 500-year wind effects at different directions. As expected, there is no obvious change either in the magnitude of predictions in each direction or in the directionality characteristics of wind effects. Therefore, the overall prediction with consideration of directionality should also not be affected by the masking issue, as shown in Table 3. It is noted that all the predictions are normalized by the maximum 500-year wind effect amongst all directions with the use of full rank data.

\section{A simplified method}

The prediction made by taking correlation into consideration should fall into the region bounded by the two variants of SBS method, i.e., SBS1 and SBS2. One can make a judgment whether the multivariate approach is needed by comparing the difference of the estimations from SBS1 and SBS2. The necessity of using multivariate approaches was well demonstrated considering the results from the previous two examples with full rank data. In the case of Example 1, the estimates from two SBS methods for $\mathrm{R}=50$ and 500 years are 0.673 and 1.000 versus 0.773 and 1.111 as shown in Table 4, resulting in differences of $14.9 \%$ and $11.1 \%$, respectively, which one may consider it to be too large to be acceptable. Therefore, a 
multivariate approach is needed for an accurate solution. However, in the case of Example 2 the differences are smaller, e.g., $0.9 \%$ and $0.0 \%$ for $\mathrm{R}=50$ and 500 years, respectively, which suggest that the solutions from multivariate approach will not make too much difference to the independent assumption and therefore it is not necessary.

Moreover, even though the multivariate approach may be needed in Example 1, it is not necessary to account for the full dependence structure of all directions because weak correlation between directions has negligible influence on the prediction and thus can be approximated as independent. That is equivalent to say that it is sufficient to only account for the dependence structures among those strongly correlated dominant directions, while the other weakly correlated directions can be regarded as independent. Also, the computational effort for the CDF multivariate Gaussian distribution increases exponentially when the number of variables increases, especially for small failure probability, i.e., large MRI (Genz and Bretz 2002). Based on the above statements, the following simplified procedure is developed to serve the purposes of accurate solutions as well as less computational efforts:

1) Estimate the JCDF $\Psi_{X, c o r r}(x)$ of $n_{\text {dom }}$ largest wind effect directions that are correlated using one of the multivariate models;

2) The directions that are not included in computing $\Psi_{X, \text { corr }}$ are considered to be independent. Their JCDF is computed as $\Psi_{X, i n d}(x)=\prod \Psi_{X_{i}}(x)$;

3) The JCDF of all directions is then calculated as $\Psi_{X, n_{d o m}}(x)=\Psi_{X, \text { ind }}(x) \cdot \Psi_{X, \text { corr }}(x)$ and the 352 corresponding R-year wind effect $x_{R, n_{d o m}}$ is determined through $\Psi_{X, n_{d o m}}\left(x_{R}\right)=1-1 / \mathrm{R}$;

4) Repeat 1) 3) with $n_{\text {dom }}+1$ largest wind effect directions, and Compare the estimations. If the difference is acceptable, end the calculation. Otherwise add more directions.

One can begin the procedure by taking the strongest two directions with strong correlation for the first trial, and then adding the third strongest wind effect direction for the second trial and so on. Because 357 the strongly correlated directions with dominant wind effects are often limited in number, the prediction can be converged and approaches the accurate solution after several iterations. 
To illustrate the simplified method, we continue with Example 1. For the first trial, let $n_{\text {dom }}=2$ and

360 the most dominant two directions, $\mathrm{W}$ and $\mathrm{WNW}$, are used and $\Psi_{X, n_{d o m}=2}$ is calculated and plotted in Fig.

7. Then for the second trial, the third strongest direction NW is added to the dominant directions and

$\Psi_{X, n_{\text {dom }}=3}$ is calculated and plotted in the same figure. One may possibly stopped after $n_{d o m}=3$ because

$\Psi_{X, n_{\text {dom }}=3}$ and $\Psi_{X, n_{\text {dom }}=2}$ are almost identical which means adding more directions to the multivariate

364 structure will not change the estimate anymore. However, for verification purpose, the estimation with all

36516 directions is also carried out. It can be seen that it is sufficient enough by considering the dependence

366 structures of two dominant directions, i.e., NW and WNW. It results in 0.749 and 1.095 for $\mathrm{R}=50$ and

367500 years, which reduced the corresponding estimates from SBS2 by $3.3 \%$ and $1.5 \%$, respectively. By the

368 way, the estimates with multivariate modeling of 16 directions are almost identical to the results with multivariate modeling of two directions.

\section{The difference between Gaussian and Gumbel copula models}

Again, we begin with bivariate variables and assumed that there are two directions with identical

372 Gumbel distributions for extreme wind speeds, e.g., $\mu_{v}=15$ and $V_{v}=0.3$, and the same wind load coefficient value is assigned for each direction. Then the difference of estimated wind effect $\delta x$ for $\mathrm{R}=$

37450 years and for flexible structures $(\mathrm{b}=3)$ is calculated at various $\rho_{i j}$ using both copula models as shown

375 in Fig. 8. It is obvious that the prediction differs with different copula used. For $\rho_{i j}=0.7$, the reductions 376 of prediction from independent assumption are $\delta x=5.4 \%$ and $12.3 \%$ for Gaussian and Gumbel copula

377 models, respectively. Moreover, if one thinks there is a minimum acceptable $\delta x$ value to consider

378 directional dependence for one particular model, e.g., a difference of $5 \%$ to be critical, then the 379 corresponding critical correlation coefficients should be different for these two models, e.g., $\rho_{i j}=0.68$ 380 for Gaussian copula model, and 0.35 for Gumbel copula model.

To further investigate the difference brought by models for more than two correlated directions, the simplified method was used when $n_{d o m}=2$ so that the most dominant four directions from Example 1 are 
included while $\Psi_{X, c o r r}(x)$ is calculated using the Gumbel copula model, and the prediction is compared

384 with that from the Gaussian copula model. Fig. 9 shows the comparison of the yearly maximum

385 distributions of wind effect. The lower bound and is represented by " $\mathrm{x}$ " marker that is calculated from

386 SBS1. The upper bound represented by "+" marker is the prediction from SBS2. The prediction from

387 Gumbel copula model (solid line with triangle marker) is slightly lower than that from Gaussian copula 388 model (solid line with square marker), which echoes the result shown in Fig. 8. The differences brought by two models are not significant, which are $1.6 \%$ and $2.1 \%$ when $b=2$, and $1.8 \%$ and $2.9 \%$ when $b=3$, for 50 and 500-year wind effects.

\section{Influence of partition of directional sectors}

In wind tunnel tests, a high resolution partition is often needed to narrows down the angular width of each directional sector in order to gain a thorough understanding of the directionality characteristics of wind loads and load effects, especially for those sensitive to the variation of direction. On the other hand, when it comes to the partition of wind speed data, depending on the available number of observations the number of directional sectors should be restricted by the allowance of model uncertainty. The coefficient of variation is inversely proportional to the square root of the number of observations in a particular direction (Isyumov et al. 2014). This is particularly true for a masked wind speed data set as the sum of the number of observations per sector equals to the length of record. However, the use of a full rank wind speed data set compensates such problem as the sample size of wind speed in each direction and regardless of direction are the same. There is a reasonable judgment to the partition of directional sectors to achieve a balance between the number of directions and model uncertainty.

The following discussion is on the comparison of partitioning the wind speed by 8 and 16 sectors 404 given that the 16-sector partition wind tunnel test were carried out with the wind load coefficient as 405 shown in Examples 1 and 2. The wind speed we used are from full rank data. Now suppose the wind 406 speed was partitioned into eight sectors centered on E, NE, ..., SE. By re-modeling the wind climate with 4078 sectors, the 500-year directional wind speeds are calculated and shown in Fig. 10. The non-directional 
wind speeds estimated from 8 -sector partition are almost the same as those from 16 -sector partition. The 50- and 500-year wind speed are 65.5 and $78.1 \mathrm{mph}$, determined from direct fit to non-directional data, and 67.9 and $81.9 \mathrm{mph}$, determined from Gaussian copula model.

As for the wind load coefficient, one may naturally pick the values corresponding to the center direction. For E, NE, ..., SE wind direction, the wind load coefficients from E, NE, ..., and N in Fig. 5 are taken as shown in Fig.11. Fig. 12 shows the 500-year wind effects at different directions. However,

414 with such a decision, the overall wind effect may be underestimated as the maximum wind effect over all 415 directions may be omitted. For instance, in Example 2, due to the omission of wind load coefficient in the other directions, the estimated 500-year wind load effect using Gaussian copula model is 0.861 as shown in Table 5 denoted as Case R0, which is $1.6 \%$ less than the result calculated with 16 -sector wind climate partition as shown in Table 4.

Isyumov et al. (2014) suggested rotating the directional wind speed within a certain angular range and the largest prediction from all cases is used for design. Such a practice not only accounts for the uncertainty of wind climate modeling but also provide a means to recover the omission of important wind load direction. Following this idea, we rotate the wind climate by -22.5 and +22.5 degrees and the wind effects are recalculated, referred to as Cases R1 and R2, respectively. As a result of such a rotation, for example, wind speed in W, corresponds to wind load coefficient at directions WSW and WNW in Cases $\mathrm{R} 1$ and R2, respectively. As shown in Table 4 and 5, when the largest predictions from Cases R0, R1 and $\mathrm{R} 2$ are chosen (as shown in bold font), the predictions become more conservative compared to Case R0.

427 For instance, in Example 1, using the 16-sector partition, the 500-year wind effect estimated with rotation 428 is 1.213 , which is $10.8 \%$ larger than the results without rotation, i.e., 1.095 in case R0. It is also noted that 429 by rotating directional wind speed model, the predictions from 8 -sector partition is much closer to the 430 results from 16-sector partition.

The wind load coefficient of a given direction can also be corrected and calculated as weighted average of itself and the ones of two neighboring directions when 8-sector wind climate model is used.

433 For instance, the wind load coefficient in $\mathrm{N}$ direction is calculated as $0.5 c_{\mathrm{N}}+0.25 c_{\mathrm{NNW}}+0.25 c_{\mathrm{NNE}}$, 
434 where, for example, $c_{N}$ is original wind effect coefficient in $\mathrm{N}$ direction. The assumption behind such

435 weighting factors is that the wind direction is evenly distributed within one sector and the center direction

436 takes half of the width of a sector while the neighboring two directions share the other half. Fig.11 shows

437 these weighted coefficients at different directions. With these weighted coefficients, calculations similar

438 to previous Cases R0, R1 and R2 are repeated as denoted as Cases R3, R4 and R5. The results are shown

439 in Table 4c. It is seen that with this correction, the estimated wind effects from 8 -sector becomes less

440 sensitive to the rotation of wind direction and may be smaller or larger than the results without corrections.

441 For Example 1, the estimates of 500-year wind effect with wind load coefficient correction are larger, i.e.,

4421.103 versus 1.095 while for Example 2, it is smaller, i.e., 1.055 versus 1.249. Therefore, the best

443 estimate is to use the maximum prediction among all cases of R0 to R6 for conservatism purpose.

Following the calculation for directional wind effect, the directionality factor can be determined as

445 the ratio of predictions with consideration of directionality to that of worst case consideration, i.e., the

446 combination of the directionless wind speeds and the maximum wind load coefficient of all sectors for

447 each case. Table 7 summarizes the directionality factor for various cases. It can be seen that the

448 directionality factor varies from case to case due to the change of directionality in wind load coefficient. It

449 is also noted that the directionality factors are not sensitive to the change of MRIs in both examples.

\section{8. Conclusion}

This study addressed a number of issues related to the modeling of multivariate distribution of 452 directional extreme wind speeds and its influence on the predicted wind effects with various MRIs. The 453 correlation of dominant directional wind speeds has a considerable effect on predicted wind effects, 454 especially, on those with lower MRIs. The influence of correlation increases when the number of 455 correlated directions increases and when the variation of wind speed is large. The influence on wind 456 effects of flexible structures is larger than that of rigid structures. 
The distributions and their predictions of wind speed and wind effect in each direction and regardless

458 of direction are not much affected by the masking issue when the maximum mean hourly wind speed is

459 reported at either 3-hour or 24-hour interval.

460 The estimation with consideration of correlation of directional wind speeds is between those from

461 two variants of sector-by-sector method where a full correlation and independence were assumed

462 respectively. A simplified procedure was proposed for engineering practice in which the correlation of

463 only a small number of directional wind speeds needs to be considered. Model difference exists between

464 Gaussian and Gumbel copula models but it is less significant.

465 The partition of directional sectors should consider the balance of model uncertainty and sensitivity 466 of directionality of wind climate, aerodynamic and structural dynamics. Considering the effects of the 467 modeling uncertainty, rotating directional wind speed in a certain range and seeking maximum response 468 can minimize the risk of underestimation. The correction to wind load coefficient using weighted average 469 of adjacent directions may be a good adjustment to the estimation with less number of directional wind 470 speed sector. The directionality factor depends on the directional characteristics of wind speed and wind 471 effect coefficient but it is not sensitive to the change of MRI.

\section{Acknowledgements}

473 The support for this work provided in part by NSF Grant No. CMMI-1029922 and CMMI-1536108

474 is greatly acknowledged.

475 


\section{References}

American Society of Civil Engineers, 2010. Minimum design loads for buildings and other structures, ASCE Standard ASCE/SEI 7-10.

Chen, X., and Huang, G. (2010). "Estimation of probabilistic extreme wind load effects: combination of aerodynamic and wind climate data." Journal of Engineering Mechanics, 136(6), 747-760.

Coles, S. G., and Walshaw, D. (1994). "Directional modelling of extreme wind speeds.” Applied Statistics, 43(1), 139-157.

Cook, N. J. (1982). “Towards better estimation of extreme winds.” Journal of Wind Engineering and Industrial Aerodynamics, 9(3), 295-323.

Cook, N. J., and Mayne, J. R. (1979). “A novel working approach to the assessment of wind loads for equivalent static design.” Journal of Wind Engineering and Industrial Aerodynamics, 4(2), 149-164.

Cook, N. J., and Mayne, J. R. (1980). “A refined working approach to the assessment of wind loads for equivalent static design.” Journal of Wind Engineering and Industrial Aerodynamics, 6, 125-137.

Davenport, A. G. (1977). “The prediction of risk under wind loading.” Proceedings of the 2nd International Conference on Structural Safety and Reliability, Munich, Germany, September, 1977, $511-538$.

Diniz, S. M. C., Sadek, F., and Simiu, E. (2004). "Wind speed estimation uncertainties: effects of climatological and micrometeorological parameters." Probabilistic Engineering Mechanics, 19(4), $361-371$.

Diniz, S. M. C., and Simiu, E. (2005). "Probabilistic descriptions of wind effects and wind-Load factors for database-assisted design." Journal of Structural Engineering, 131(3), 507-516. 
497

Genz, A., and Bretz, F. (2002). "Comparison of methods for the computation of multivariate $t$ probabilities." Journal of Computational \& Graphical Statistics, 11(4), 950-971.

Grigoriu, M. (2007). "Multivariate distributions with specified marginals: applications to wind engineering." Journal of Engineering Mechanics, 133(2), 174-184.

Grigoriu, M. (2009). “Algorithms for generating large sets of synthetic directional wind speed data for hurricane, thunderstorm, and synoptic winds." NIST Technical Note, 1626.

Hanzlik, P., Diniz, S., Grazini, A., Grigoriu, M., and Simiu, E. (2005). "Building orientation and wind effects estimation.” Journal of Engineering Mechanics, 131(3), 254-258.

Haraguchi, K., and Kanda, J. (1999). "Probability model for annual maximum wind speeds in multidirection." Wind Engineering into the 21st Century, ICWE, Larsen, Larose \& Livesey, Balkema, $205-212$.

Harris, R. I. (1982). “An improved method for the prediction of extreme values of wind effects on simple buildings and structures.” Journal of Wind Engineering and Industrial Aerodynamics, 9(3), 343-379.

Harris, R. I. (2005). "A new direct version of the Cook-Mayne method for wind pressure probabilities in temperate storms." Journal of Wind Engineering and Industrial Aerodynamics, 93(7), 581-600.

Holmes, J. (2001). Wind Loading of Structures. CRC Press.

Irwin, P. A., Garber, J., and Ho, E. (2005). "Integration of wind tunnel data with full scale wind climate." Proceedings of the10th Americas Conference on Wind Engineering, Baton Rouge, Louisiana, U.S.A, May 31 - June 4, 132-135.

Isyumov, N., Ho, E., and Case, P. (2014). "Influence of wind directionality on wind loads and responses.” Journal of Wind Engineering and Industrial Aerodynamics, Elsevier, 133, 169-180. 
518

519

520

521

522

523

524

525

526

527

528

529

530

531

532

533

534

535

536

537

Isyumov, N., Mikitiuk, M., Case, P., Lythe, G., and Welburn., A. (2003). "Predictions of wind loads and responses from simulated tropical storm passages." Proceedings of the 11th International Conference on Wind Engineering, Lubbock, Texas, Jun, 2003, CD-ROM.

Itoi, T., and Kanda, J. (2002). "Comparison of correlated Gumbel probability models for directional maximum wind speeds." Journal of Wind Engineering and Industrial Aerodynamics, 90(12-15), $1631-1644$.

Kareem, A. (1987). “Wind effects on structures: a probabilistic viewpoint.” Probabilistic Engineering Mechanics, 2(4), 166-200.

Kareem, A. (1988). “Aerodynamic response of structures with parametric uncertainties.” Structural Safety, 5(3), 205-225.

Kareem, A. (1990). "Reliability analysis of wind-sensitive structures." Journal of Wind Engineering and Industrial Aerodynamics, 33(3), 495-514.

Lepage, M. F., and Irwin, P. A. (1985). “A technique for combining historical wind data with wind tunnel tests to predict extreme wind loads." Proceedings of the 5th U.S. National Conference on Wind Engineering, Lubbock, Texas, November, 1985, 6-8.

Mayne, J. R. (1979). “The estimation of extreme winds.” Journal of Wind Engineering and Industrial Aerodynamics, 5(1-2), 109-137.

Moriarty, W. W., and Templeton, J. I. (1983). “On the estimation of extreme wind gusts by direction sector." journal of w, 13, 127-138.

Nelsen, R. B. (2006). An introduction to copulas. Springer, New York, NY. 
Payer, T., and Küchenhoff, H. (2004). "Modelling extreme wind speeds at a German weather station as basic input for a subsequent risk analysis for high-speed trains." Journal of Wind Engineering and Industrial Aerodynamics, 92(3-4), 241-261.

Rigato, A., Chang, P., and Simiu, E. (2001). "Database-assisted design, standardization, and wind direction effects." Journal of Structural Engineering, 127(8), 855-860.

Sadek, F. (2005). Federal Building and Fire Safety Investigation of the World Trade Center Disaster: Baseline Structural Performance and Aircraft Impact Damage Analysis of the World Trade Center Towers. NIST, NCSTAR 1-2.

Schweizer, B., and Sklar, A. (1983). Probabilistic Metric Spaces. North-Holland, New York.

Simiu, E., and Filliben, J. J. (1981). "Wind direction effects on cladding and structural loads." Engineering Structures, 3, 181-186.

Simiu, E., and Filliben, J. J. (2005). "Wind tunnel testing and the sector-by-sector approach to wind directionality effects.” Journal of Structural Engineering, 131(7), 1143-1145.

Simiu, E., and Heckert, N. A. (1998). "Ultimate wind loads and direction effects in non-hurricane and hurricane-prone regions." Environmetrics, 9(4), 433-444.

Simiu, E., Hendrickson, E. M., Nolan, W. A., Olkin, I., and Spiegelman, C. H. (1985). "Multivariate distributions of directional wind speeds.” Journal of Structural Engineering, 111(4), 939-943.

Vega-Avila, R. E. (2008). “Wind directionality: a reliability-based approach.” Ph.D. dissertation, Texas Tech University, 2008

Yeo, D. (2014). "Generation of large directional wind speed data sets for estimation of wind effects with long return periods.” Journal of Structural Engineering, 140(10), 04014073. 
559 Zhang, X., and Chen, X. (2015). "Assessing probabilistic wind load effects via a multivariate extreme

560 wind speed model: A unified framework to consider directionality and uncertainty." Journal of Wind

$561 \quad$ Engineering and Industrial Aerodynamics, http://dx.doi.org/10.1016/j.jweia.2015.09.002.

562 
564

565

Table. 1. The covariance matrix of Gaussian variables from full rank data

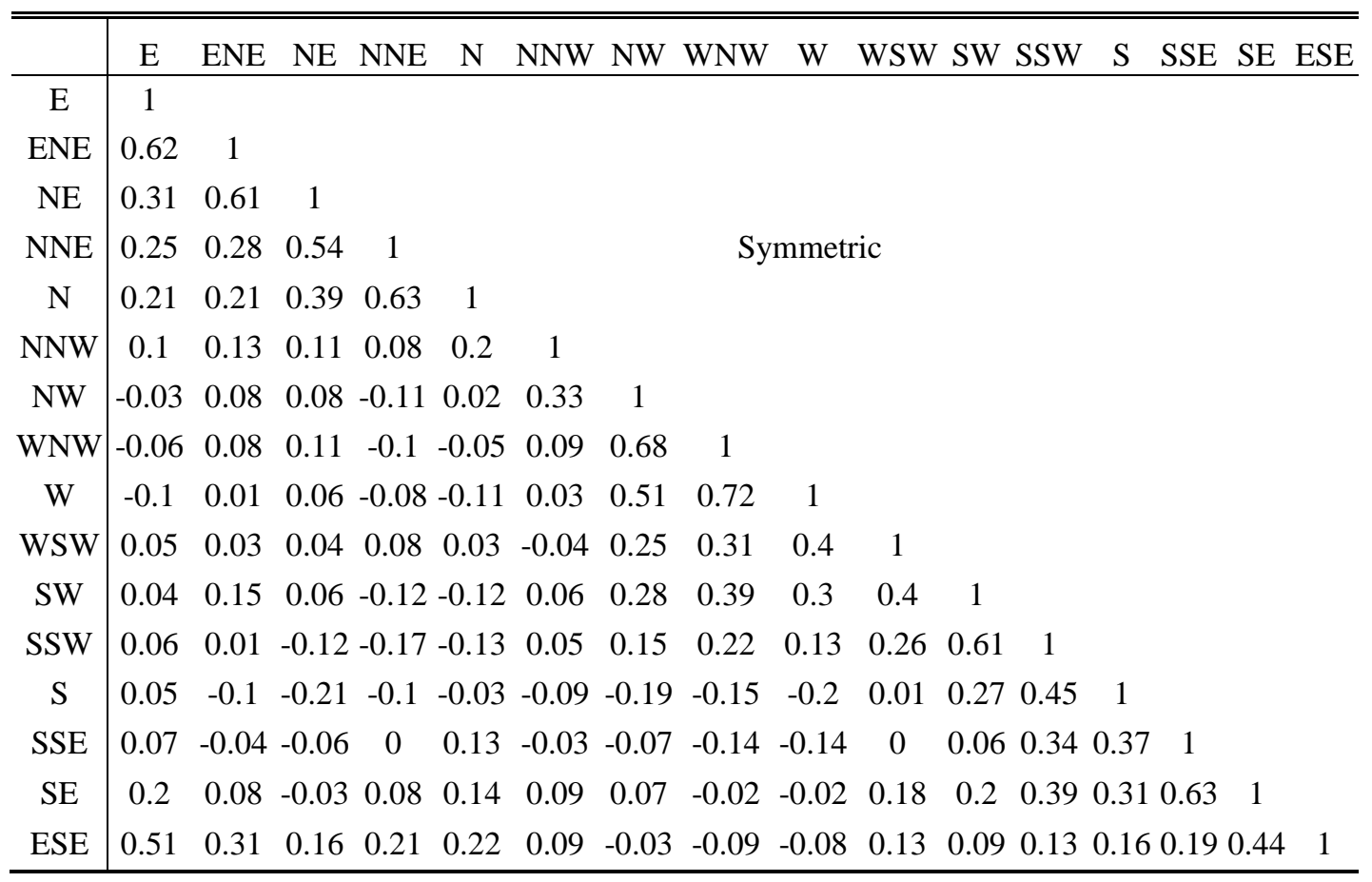


Table 2. Predicted 50- and 500-year non-directional wind speeds (mph)

\begin{tabular}{ccccc}
\hline $\begin{array}{c}\text { MRI } \\
\text { (Year) }\end{array}$ & Data & $\begin{array}{c}\text { Full } \\
\text { rank }\end{array}$ & $\begin{array}{c}\text { 3-hour } \\
\text { interval }\end{array}$ & $\begin{array}{c}\text { 24-hour } \\
\text { interval }\end{array}$ \\
\hline 50 & 65.5 & 69.9 & 68.4 & 69.8 \\
500 & 78.1 & 84.8 & 82.2 & 84.7 \\
\hline
\end{tabular}

570

571 
Table 3. Predicted 50- and 500-year extreme wind load effects

\begin{tabular}{lcccc}
\hline \hline & $\begin{array}{c}\text { MRI } \\
\text { (Year) }\end{array}$ & $\begin{array}{c}\text { Full } \\
\text { rank }\end{array}$ & $\begin{array}{c}\text { 3-hour } \\
\text { interval }\end{array}$ & $\begin{array}{c}\text { 24-hour } \\
\text { interval }\end{array}$ \\
\hline Example 1 & 50 & 0.749 & 0.748 & 0.752 \\
& 500 & 1.095 & 1.065 & 1.100 \\
Example 2 & 50 & 0.585 & 0.568 & 0.591 \\
& 500 & 0.875 & 0.848 & 0.880 \\
\hline
\end{tabular}

575

576 
577

578

Table 4. Normalized directionless wind effect estimation for 16-sector partition

\begin{tabular}{|c|c|c|c|c|c|c|c|c|c|c|}
\hline & \multirow{2}{*}{$\begin{array}{l}\text { MRI } \\
\text { (Year) }\end{array}$} & \multicolumn{3}{|c|}{ 16-sector (R0) } & \multicolumn{3}{|c|}{ 16-sector (R1) } & \multicolumn{3}{|c|}{ 16-sector (R2) } \\
\hline & & SBS1 & SBS2 & $\begin{array}{c}\text { Gaussian } \\
\text { Copula }\end{array}$ & SBS1 & SBS2 & $\begin{array}{c}\text { Gaussian } \\
\text { Copula }\end{array}$ & SBS1 & SBS2 & $\begin{array}{c}\text { Gaussian } \\
\text { Copula }\end{array}$ \\
\hline \multirow[t]{2}{*}{ Example 1} & 50 & 0.673 & 0.773 & 0.749 & 0.629 & 0.713 & 0.692 & 0.759 & 0.851 & 0.827 \\
\hline & 500 & 1.000 & 1.111 & 1.095 & 0.943 & 1.028 & 1.013 & 1.113 & 1.230 & 1.213 \\
\hline \multirow[t]{2}{*}{ Example 2} & 50 & 0.582 & 0.587 & 0.585 & 0.844 & 0.854 & 0.848 & 0.353 & 0.385 & 0.380 \\
\hline & 500 & 0.874 & 0.876 & 0.875 & 1.265 & 1.270 & 1.266 & 0.521 & 0.551 & 0.550 \\
\hline
\end{tabular}

579

580

581 
583 Table 5. Normalized directionless wind effect estimation for 8-sector partition, case R0, R1 and R2

\begin{tabular}{|c|c|c|c|c|c|c|c|c|c|c|}
\hline & \multirow{3}{*}{$\begin{array}{c}\text { MRI } \\
\text { (Year) }\end{array}$} & \multicolumn{3}{|c|}{8 -sector (R0) } & \multicolumn{3}{|c|}{ 8-sector (R1) } & \multicolumn{3}{|c|}{ 8-sector (R2) } \\
\hline & & & & Gaussian & & & Gaussian & & & Gaussian \\
\hline & & SBS1 & SBS2 & Copula & SBS1 & SBS2 & Copula & SBS1 & SBS2 & Copula \\
\hline \multirow[t]{2}{*}{ Example 1} & 50 & 0.671 & 0.746 & 0.724 & 0.633 & 0.686 & 0.666 & 0.740 & 0.759 & 0.748 \\
\hline & 500 & 0.987 & 1.069 & 1.052 & 0.931 & 0.983 & 0.968 & 1.088 & 1.102 & 1.095 \\
\hline \multirow[t]{2}{*}{ Example 2} & 50 & 0.583 & 0.586 & 0.585 & 0.849 & 0.849 & 0.849 & 0.353 & 0.376 & 0.373 \\
\hline & 500 & 0.861 & 0.861 & 0.861 & 1.249 & 1.249 & 1.249 & 0.556 & 0.538 & 0.537 \\
\hline
\end{tabular}

584

585 
587 Table 6. Normalized directionless wind effect estimation for 8-sector partition, case R3, R4 and R5

\begin{tabular}{|c|c|c|c|c|c|c|c|c|c|c|}
\hline & \multirow{3}{*}{$\begin{array}{c}\text { MRI } \\
\text { (Year) }\end{array}$} & \multicolumn{3}{|c|}{ 8-sector (R3) } & \multicolumn{3}{|c|}{ 8-sector (R4) } & \multicolumn{3}{|c|}{ 8-sector (R5) } \\
\hline & & & & Gaussian & & & Gaussian & & & Gaussian \\
\hline & & SBS1 & SBS2 & Copula & SBS1 & SBS2 & Copula & SBS1 & SBS2 & Copula \\
\hline \multirow[t]{2}{*}{ Example 1} & 50 & 0.679 & 0.729 & 0.710 & 0.575 & 0.652 & 0.634 & 0.747 & 0.762 & 0.753 \\
\hline & 500 & 0.998 & 1.048 & 1.033 & 0.846 & 0.933 & 0.917 & 1.097 & 1.109 & 1.103 \\
\hline \multirow[t]{2}{*}{ Example 2} & 50 & 0.580 & 0.580 & 0.580 & 0.717 & 0.717 & 0.717 & 0.350 & 0.373 & 0.370 \\
\hline & 500 & 0.852 & 0.849 & 0.849 & 1.055 & 1.055 & 1.055 & 0.517 & 0.534 & 0.532 \\
\hline
\end{tabular}

588

589

590

591

592

593 
596

597

Table 7. Directionality factor for various cases

\begin{tabular}{|c|c|c|c|c|c|c|c|c|c|c|}
\hline & \multirow{2}{*}{$\begin{array}{c}\text { MRI } \\
\text { (Year) }\end{array}$} & \multicolumn{3}{|c|}{ 16-sector } & \multicolumn{6}{|c|}{ 8-sector } \\
\hline & & $\mathrm{R} 0$ & $\mathrm{R} 1$ & $\mathrm{R} 2$ & $\mathrm{R} 0$ & $\mathrm{R} 1$ & $\mathrm{R} 2$ & R3 & $\mathrm{R} 4$ & $\mathrm{R} 5$ \\
\hline Example 1 & 50 & 0.824 & 0.761 & 0.911 & 0.814 & 0.745 & 0.836 & 0.859 & 0.766 & 0.911 \\
\hline \multirow{3}{*}{ Example 2} & 500 & 0.818 & 0.76 & 0.91 & 0.805 & 0.736 & 0.832 & 0.851 & 0.755 & 0.908 \\
\hline & 50 & 0.646 & 0.936 & 0.42 & 0.938 & 0.938 & 0.412 & 0.938 & 0.938 & 0.484 \\
\hline & 500 & 0.659 & 0.953 & 0.414 & 0.938 & 0.939 & 0.402 & 0.938 & 0.938 & 0.474 \\
\hline
\end{tabular}

598 


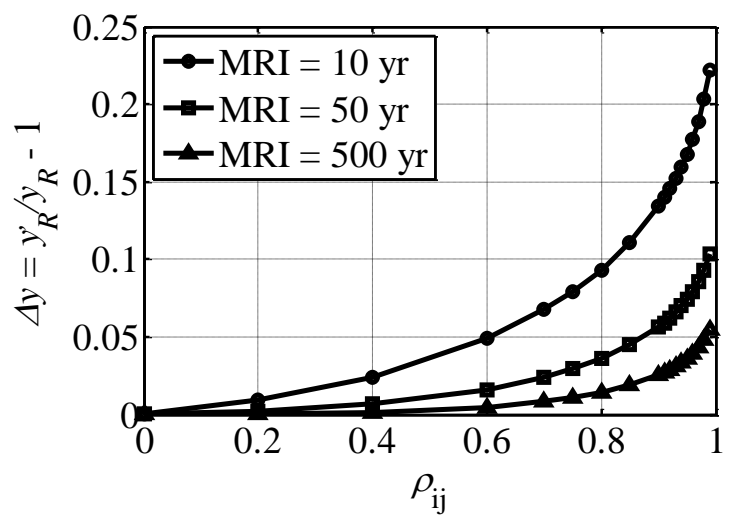

599

Fig. 1. Influence of correlation on Gaussian variables with MRI $=10,50$ and 500 years

600 
601

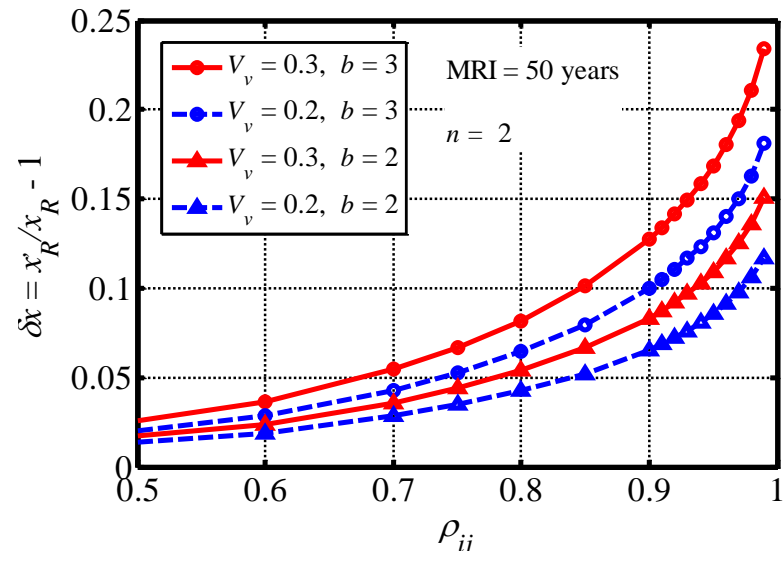

(a) $\mathrm{MRI}=50$ years

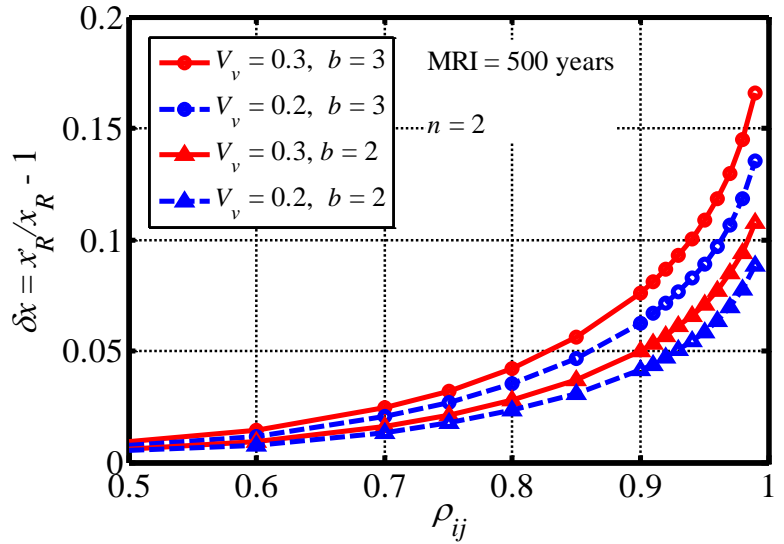

(b) $\mathrm{MRI}=500$ years 


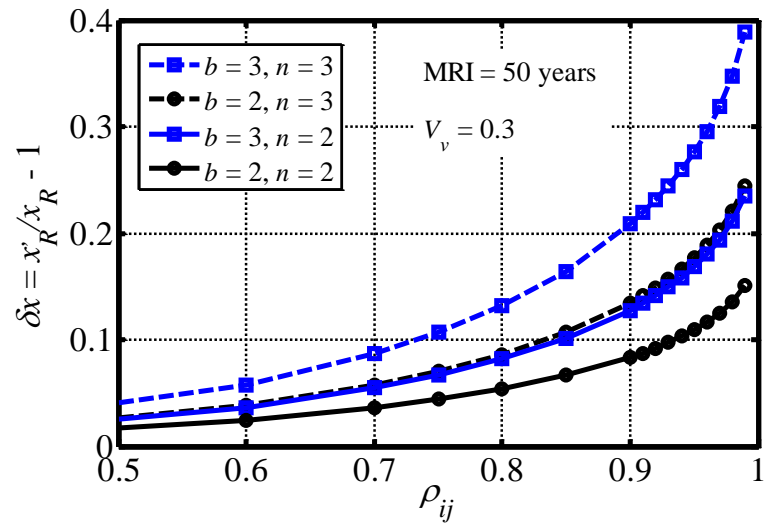

(a) $\mathrm{MRI}=50$ years

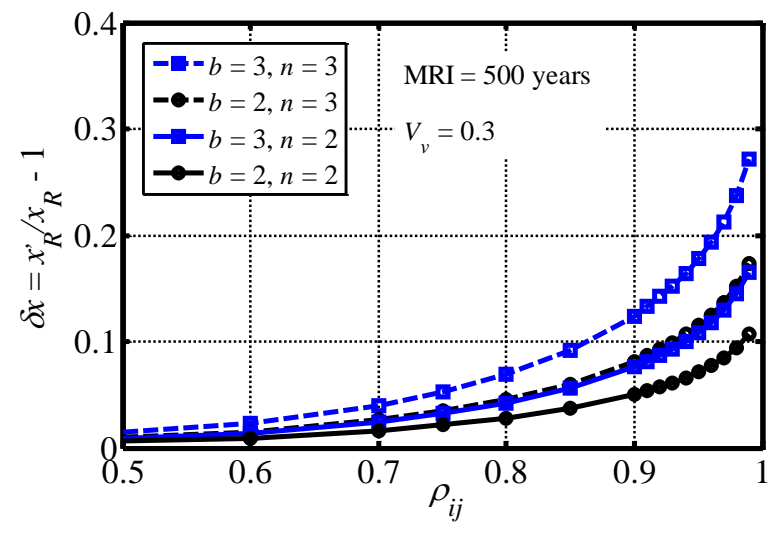

(b) $\mathrm{MRI}=500$ years
606

607

608

609
Fig. 3. Influence of number of correlated directions on the difference between wind effect calculated with and without considering correlation $\left(V_{v}=0.3\right)$ 


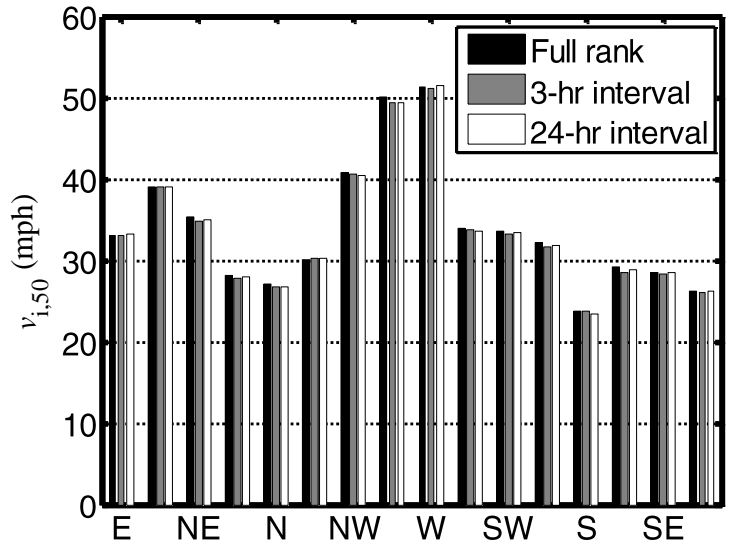

(a) MRI $=50$ years

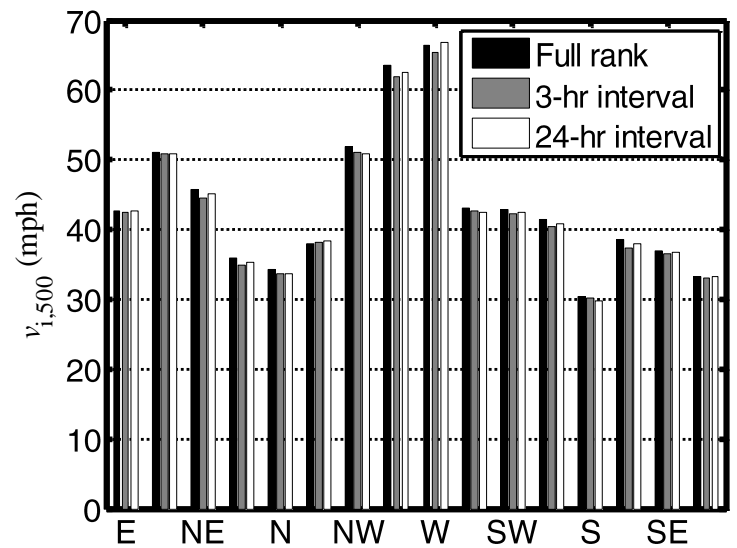

(b) MRI $=500$ years
611 612

Fig. 4. Comparison of 50- and 500-year wind speeds in each direction calculated using full rank and masked data 
614
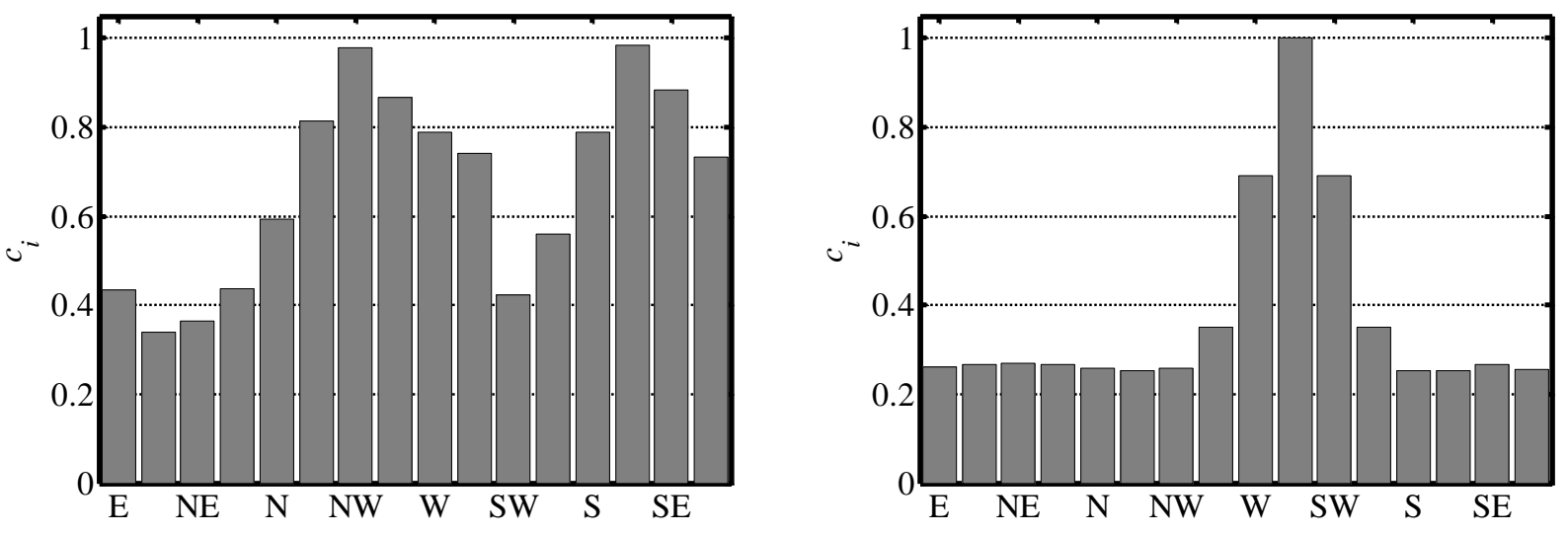

(a) Example 1

(b) Example 2

615

Fig. 5. Two examples of extreme wind load effect coefficient as a function of direction

616 


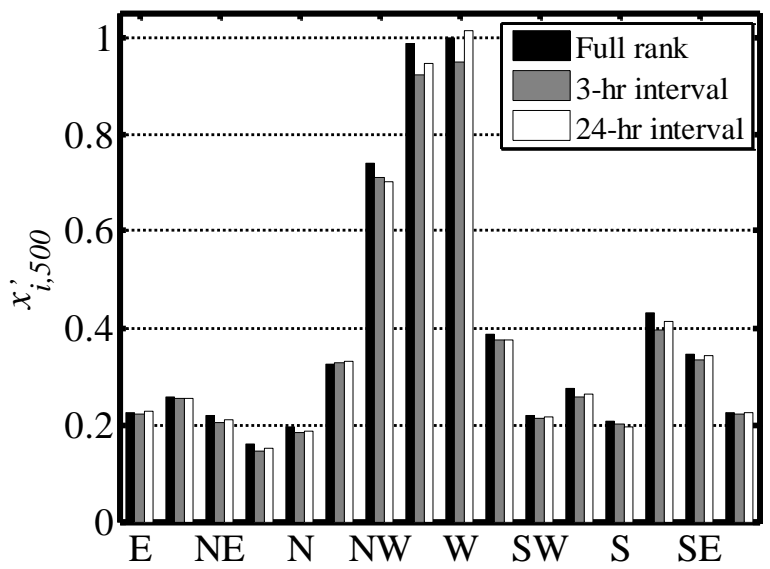

(a) Example 1

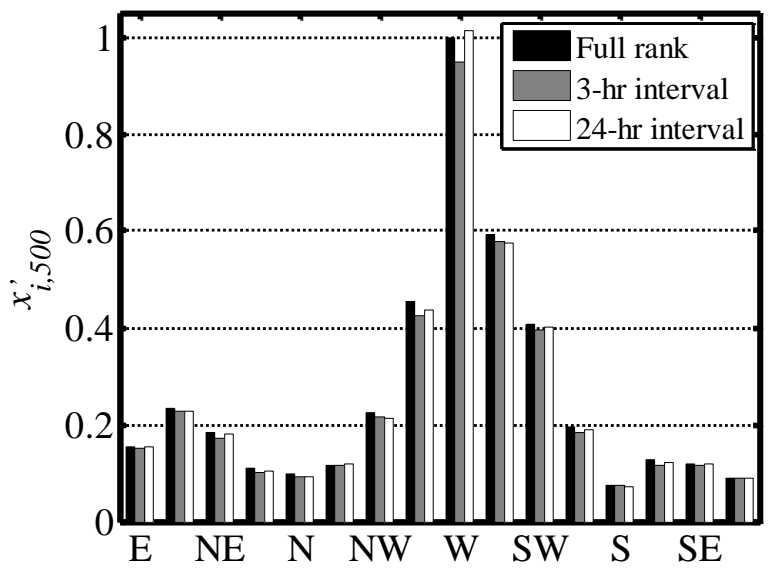

(b) Example 2

Fig. 6. 500-year extreme wind effects at different wind directions affected by wind speed masking 


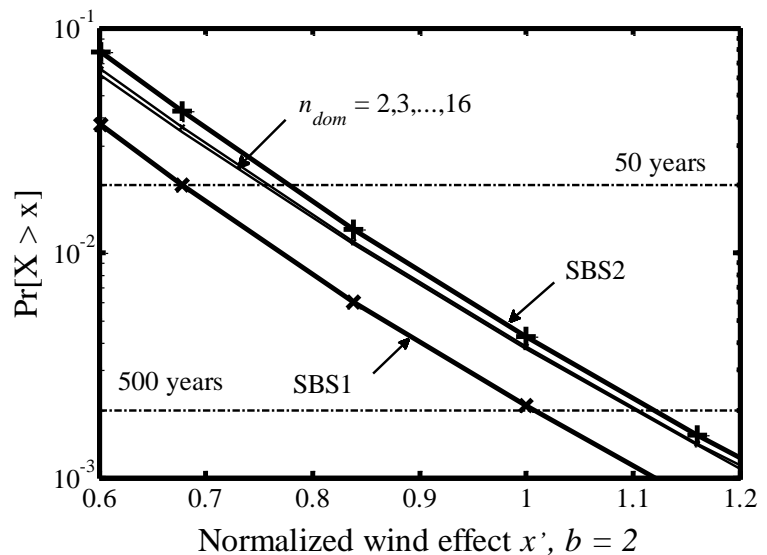

Fig. 7. Illustration of simplified procedures to calculate the distributions of yearly maximum wind effect (Example 1, b = 2) 


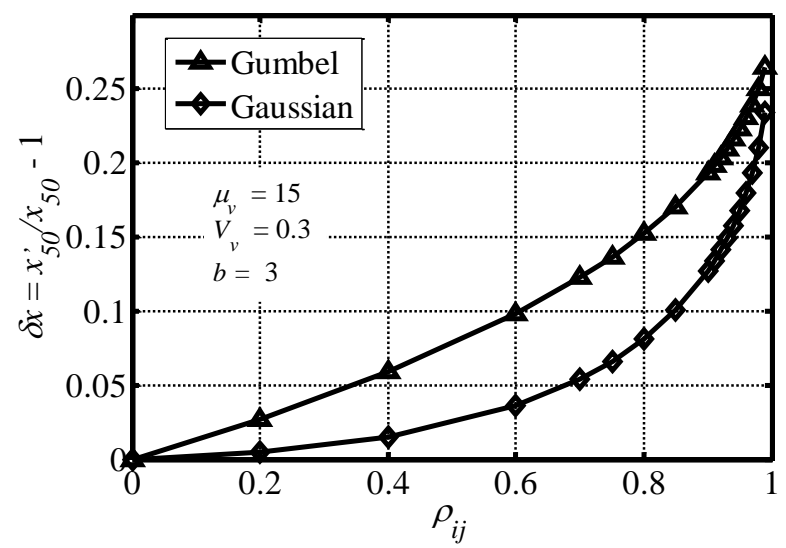

Fig. 8. Comparison of estimated 50-year wind effects using different models 


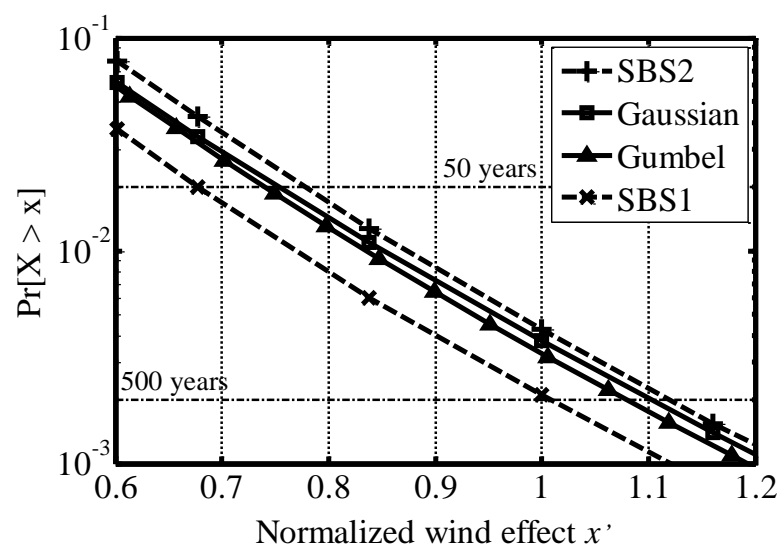

(a) $b=2$

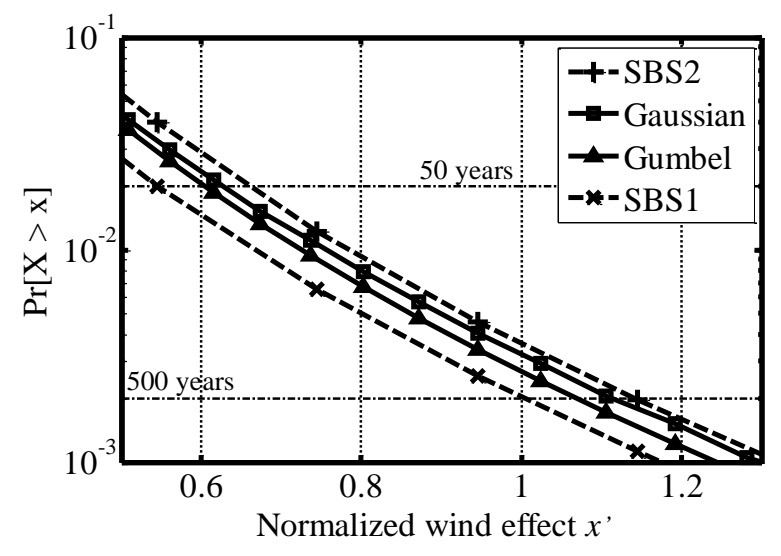

(b) $b=3$

Fig. 9. Comparison of distribution of wind effects predicted from Gaussian copula and HK model (Example 1) 


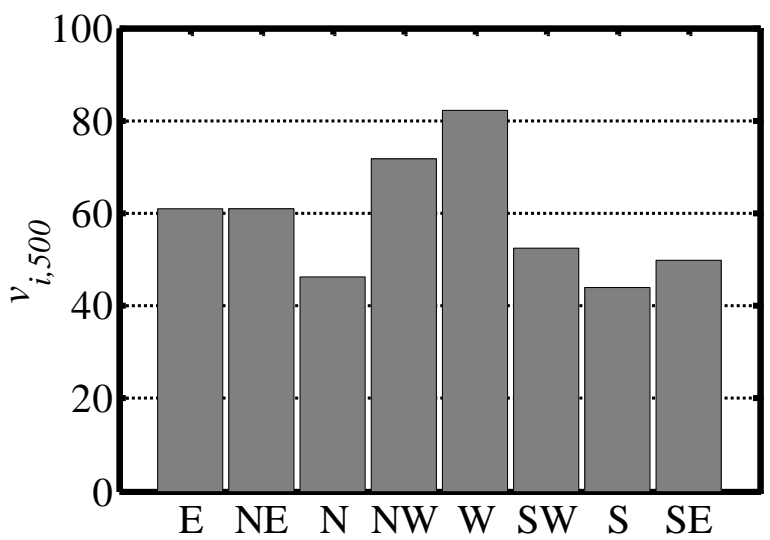

Fig. 10. 500-year wind speeds as function of wind direction affected by data masking (8-sector wind speed partition) 


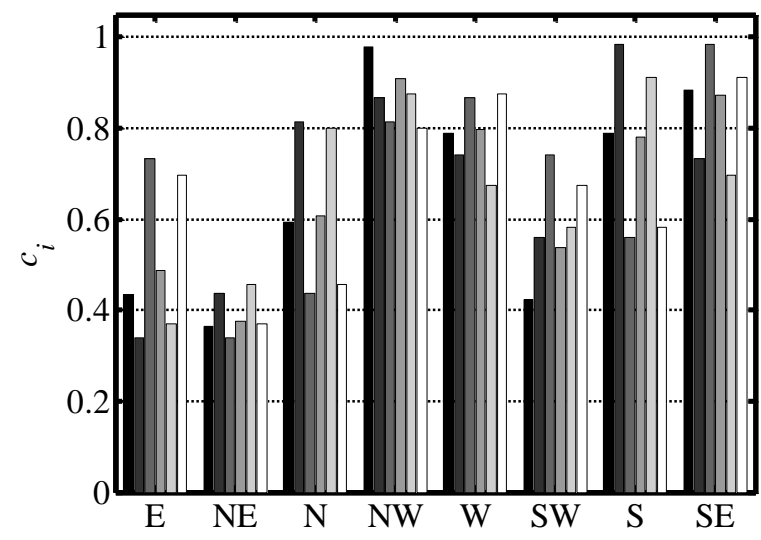

(a) Example 1

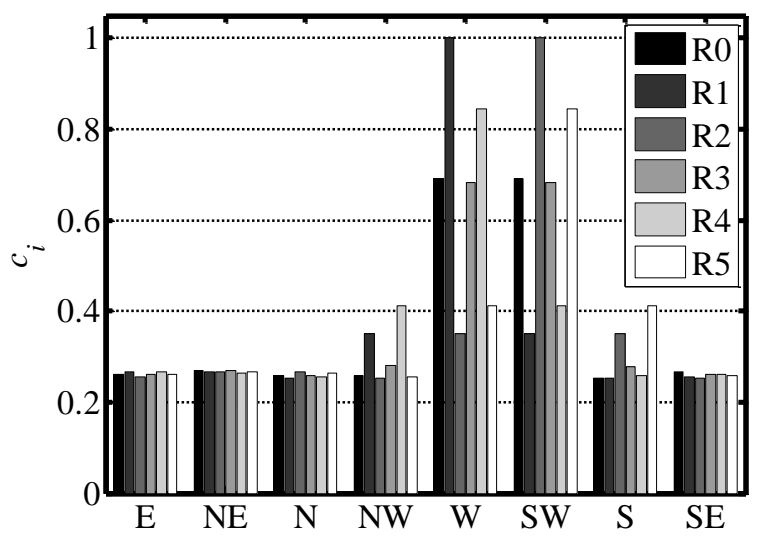

(b) Example 2

Fig.11. Wind load coefficient in each wind speed direction for 8-sector partition 


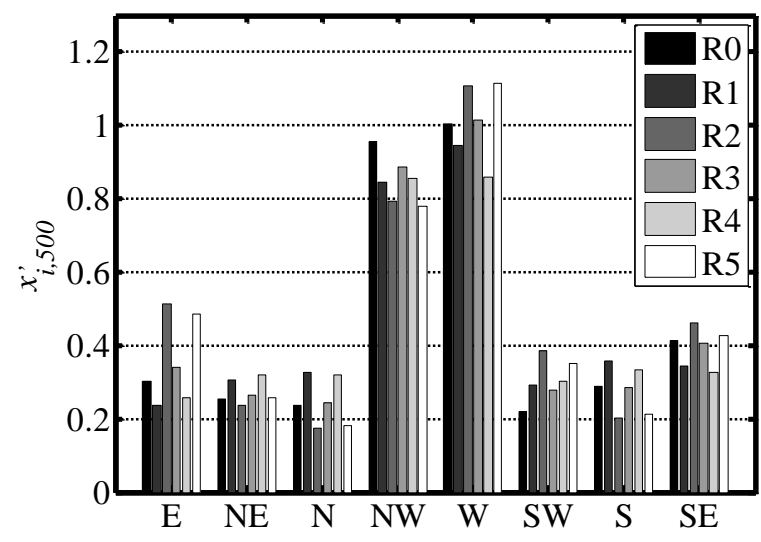

(a) Example 1

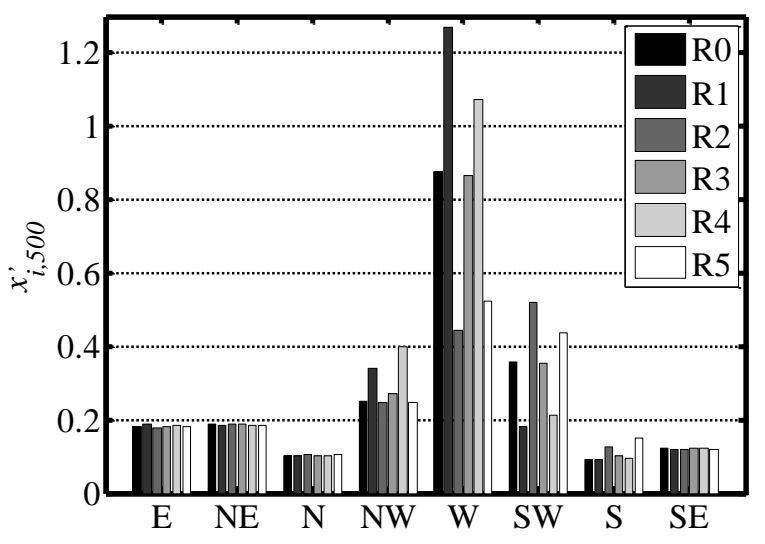

(b) Example 2

Fig. 12. Wind effect estimated in each direction for various rotations (8-sector wind speed partition) 